\title{
The environmental, socioeconomic, and health impacts of woodfuel value chains in Sub-Saharan Africa: a systematic map
}

\author{
Phosiso Sola ${ }^{1 *} \mathbb{C}$, Paolo Omar Cerutti ${ }^{2}$, Wen Zhou², Denis Gautier ${ }^{3}$, Miyuki liyama ${ }^{1,4}$, Jolien Schure ${ }^{5}$, \\ Audrey Chenevoy ${ }^{1}$, Jummai Yila ${ }^{6}$, Vanessa Dufe ${ }^{7}$, Robert Nasi ${ }^{2}$, Gillian Petrokofsky ${ }^{8}$ and Gill Shepherd ${ }^{9}$
}

\begin{abstract}
Background: In Sub-Saharan Africa (SSA), the production and use of woodfuel remains an important socio-economic activity with more than $70 \%$ of the population relying on woodfuel as their primary household energy source. Despite their socio-economic significance, woodfuel value chains are often viewed negatively due to their association with detrimental health and environmental impacts. However, the lack of sound evidence and limited understanding of the role of contextual factors in influencing the various impacts of woodfuel value chains have prevented the formulation of properly guided policy interventions. Thus the objective of this systematic map is to provide a comprehensive review of the environmental, socio-economic, and health impacts of woodfuel value chains across SSA.

Methods: The search strategy for this review map was defined in a peer-reviewed protocol and refined by iterative testing. Search strings were composed of population, intervention, and location terms and combined using Boolean operators. The bibliographic databases Web of Science, Scopus, and CAB Abstracts were used as the main sources of literature for this review, and a total of 4728 results were initially retrieved. Following title and abstract screening, 659 entered full text screening. Critical appraisal of 219 articles led to the exclusion of studies that did not set meet quality criteria for this map, resulting in a final total of 131 articles for inclusion in data extraction and analysis.
\end{abstract}

Results: From the 131 included articles, 152 individual studies were identified during data extraction. Studies came from 26 of the 49 Sub Saharan African countries, with a particular preponderance of articles published in the last 10 years. Critical appraisal found significant weaknesses in the experimental design of woodfuel value chain studies with the exception of health impact studies, which frequently utilized controls or other relevant comparators. Findings suggest that woodfuel value chains have environmental, socioeconomic and health consequences with the frequent presence of trade-offs. The reporting of contextual factors in the studies challenge the widespread perception of deforestation as being directly caused by bush fires, overgrazing and woodcutting. Instead, agricultural expansion (which often includes forest clearing) and pre-existing biophysical factors were the most frequently cited factors in shaping environmental outcomes.

Conclusions: This systematic map suggests that there are environmental, socioeconomic and health consequences associated with woodfuel value chains in Sub-Saharan Africa. However, the literature also shows a weak and geographically limited evidence base to justify the above claims. We argue that policy formulation processes targeting woodfuels in SSA require more solid, coherent and broad body of knowledge, especially for such a vital sector in rural economies. Thus, there is an urgent need to design and undertake research using robust methodologies, at

\footnotetext{
*Correspondence: p.sola@cgiar.org

${ }^{1}$ World Agroforestry Centre (ICRAF), United Nations Avenue, Gigiri, PO

Box 30677, Nairobi 00100, Kenya

Full list of author information is available at the end of the article
} and indicate if changes were made. The Creative Commons Public Domain Dedication waiver (http://creativecommons.org/ publicdomain/zero/1.0/) applies to the data made available in this article, unless otherwise stated. 
appropriate scales that further takes into account the interrelationships between environmental and socio-economic outcomes in order to generate substantial and reliable evidence for informed policy formulation.

Keywords: Charcoal, Firewood, Fuelwood, Forests, Woodlands, Livelihoods, Consumption, Production, Trade, Africa

\section{Background}

The production and use of woodfuel, defined here as encompassing firewood and charcoal, is an important socio-economic activity in Sub-Saharan Africa (SSA) with additional environmental and health consequences. Over $70 \%$ of the population relies on woodfuel as their primary household energy source, with an average per capita consumption of $0.69 \mathrm{~m}^{3} /$ year in 2011 , or 2.5 times the global average [1-3].

Although rural households mostly use firewood for cooking and heating and small-scale enterprises for manufacturing and processing (i.e. brickmaking, bread-making, tea and tobacco processing), charcoal is the dominant energy source in urban centres [4]. In comparison to firewood, charcoal has higher energy content, produces less smoke during cooking, and is easier to transport and store [3, 5]). With increasing urbanization in SSA, the demand for and use of charcoal has been projected to increase in the coming decades as long as it remains the most readily available source of energy, as well as the most affordable alternative to kerosene, liquid petroleum gas (LPG), and electricity [6, 7]. The value of the charcoal market is predicted to grow to well over US\$12 billion by 2030 [8] and the charcoal industry has been estimated to provide employment for over 10 million people across its value chain $[8,9]$.

Despite its socio-economic significance, woodfuel is often negatively portrayed, notably due to its association with negative environmental and health impacts. In SSA, wide dependence on woodfuel harvested from forests and woodlands could significantly deplete these natural resources. In addition woodfuel consumption using inefficient stoves has been said to be responsible for much of the region's total household greenhouse gas emissions [5, $10,11]$. Furthermore, indoor pollution caused by woodfuels burnt in inefficient stoves and poorly ventilated kitchens is often said to be a major cause of respiratory diseases $[5,10,12,13]$. There are complex relations between the various woodfuel value chain interventions, which comprise supply and demand activities. These activities include tree management or production; wood harvesting and processing, firewood and charcoal transportation, marketing and consumption in both rural areas and urban centres. However, most of the existing literature examines environmental, socioeconomic, or health impacts from a sector-specific perspective. Thus inter-relationship of these factors have been under-examined.
To shed light on such interrelated impacts, a team from the Center of International Forestry Research (CIFOR) and World Agroforestry Centre (ICRAF) held an inception workshop in March 2013 to brainstorm research questions on the subject and produced a draft framework with an aim of undertaking a systematic review. From the original 13 the team was expanded to 23 researchers who were organised in three groups to continue formulating review questions around woodfuel demand, supply and policy and legal frameworks. It is some members of this team and other new members that produced and published the systematic map protocol as well as revise the conceptual framework to emphasize links between supply and demand aspects of woodfuel value chains. The framework recognizes that there are a number of contextual factors that may influence environmental, socioeconomic, and health impacts: woodfuel policy frameworks, the socio-economic status of the populations involved, as well as the type and baseline conditions of the ecosystem being exploited (Fig. 1) [14]. This framework also suggests that it is the actions of these actors that bring about environmental (degradation, regeneration, carbon emissions etc.), socio-economic (employment, income, assets etc.), and health impacts (pollution, illness etc.).

Once the protocol was published, 11 of the 23 team members undertook literature screening, quality assessment, and preliminary data extraction for the systematic map. The rest of team reviewed and provided advice on the methodologies used by the team. This article presents a detailed documentation of the process used to produce the systematic map and the map itself, highlighting the quantity and quality of current status of knowledge on the environmental, socioeconomic, and health impacts of woodfuel value chains in SSA.

\section{Objective of the review}

The lack of sound evidence and limited understanding of the role of contextual factors influencing the environmental, socio-economic, and health impacts of woodfuel value chain prevent properly guided policy interventions [14, 15]. Thus the objective of this systematic map is to provide a balanced and comprehensive review of the literature on the role and impacts of woodfuel value chains across SSA. The primary review question is: "What are the environmental, socio-economic, and health impacts of woodfuel supply and demand in Sub-Saharan Africa?" This article thus presents the best available evidence of woodfuel 


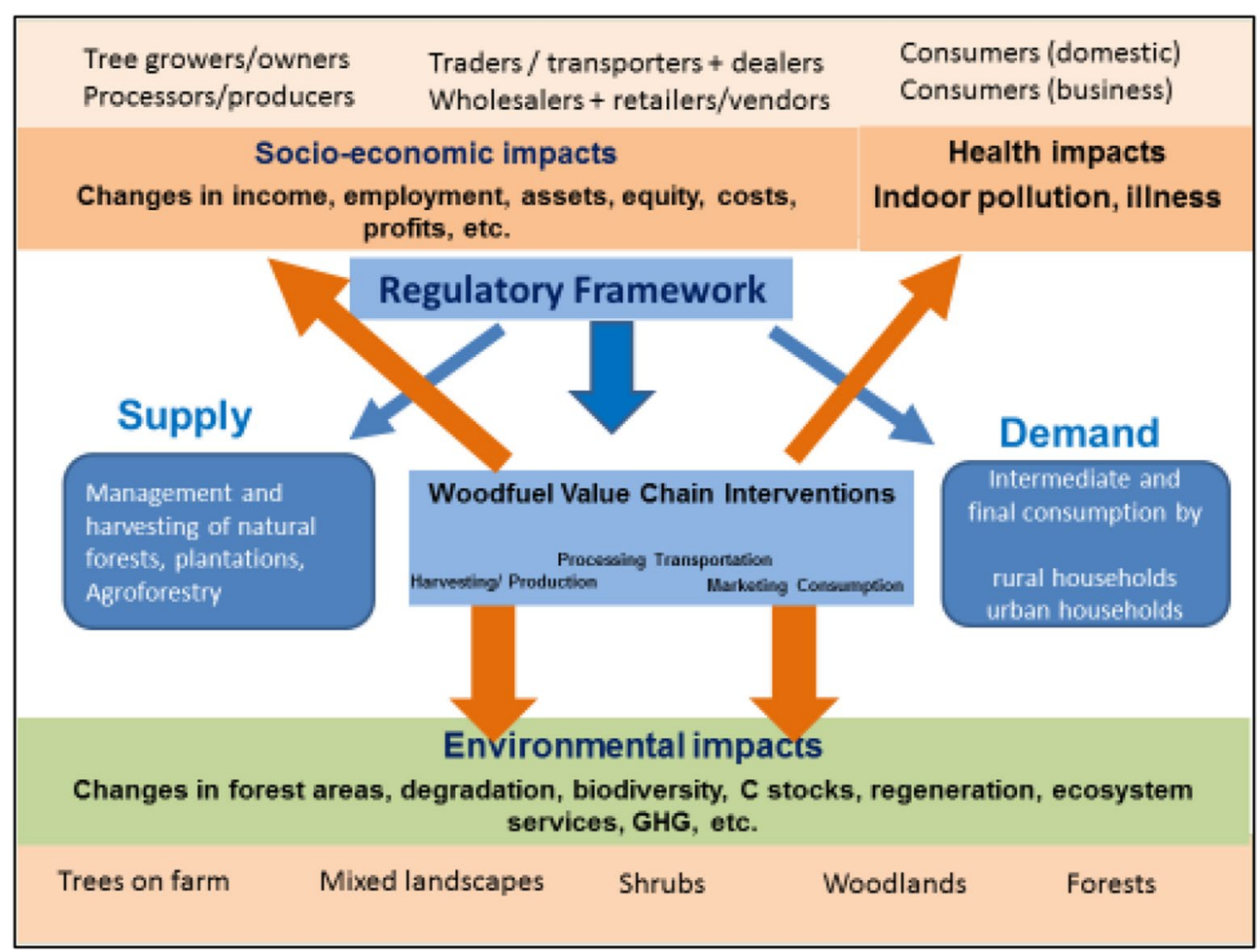

Fig. 1 Conceptual framework

value chain impacts in SSA to guide recommendations for policy and further research.

\section{Methods}

\section{Search strategy}

The search strategy for this review was defined in a peer-reviewed protocol [14] and refined by iterative testing on Web of Science and CAB Abstracts. Search strings were composed of population, intervention, and location terms and combined using Boolean operators. The bibliographic databases of Web of Science, Scopus, and $C A B$ Abstracts were used as the main sources of literature for this review, and the search strings used in each of these databases are attached in Annexure 1. Electronic searching took place in January 2015, and no language limits were placed on the search to allow for the capture of relevant foreign language studies with English abstracts. The search did not include the additional specialist websites and internet search engines defined in our protocol due to time constraints. This led to inclusion of fewer grey literature references than might have otherwise been the case, especially literature from the 1980 and 1990s which were generated before the internet was fully operational. However, in order to ensure that key references were captured in our review, we circulated a bibliography of full text screening results among the review team composed of subject specialists for further identification of key missing references for inclusion.

\section{Study screening and inclusion criteria}

Screening of studies was conducted in two stages. First, simultaneous title and abstract screening of 3979 studies was conducted using Abstrackr software. Abstrackr is a free online tool into which review teams can upload citation databases to divide abstract screening among a set number of reviewers, and from which a final list of included studies can be exported at the end of the screening process [17]. Prior to Abstrackr screening four reviewers conducted pilot screening rounds of 25 articles each, for which Randolph's free-marginal multirater kappa statistic was calculated [16]. Disagreements in screening decisions were discussed in order to reach a common understanding of the application of the inclusion criteria. Once the kappa statistic reached 0.63 , reviewers proceeded to conduct title and abstract screening individually. The abstract screening criteria is presented in Table 1.

The second stage was full text screening. This was also preceded by several rounds of pilot screening by the larger review team. However, the kappa analysis of these pilot results showed that there was only sufficient concurrence in screening between WZ, PS, VD, POC, and 


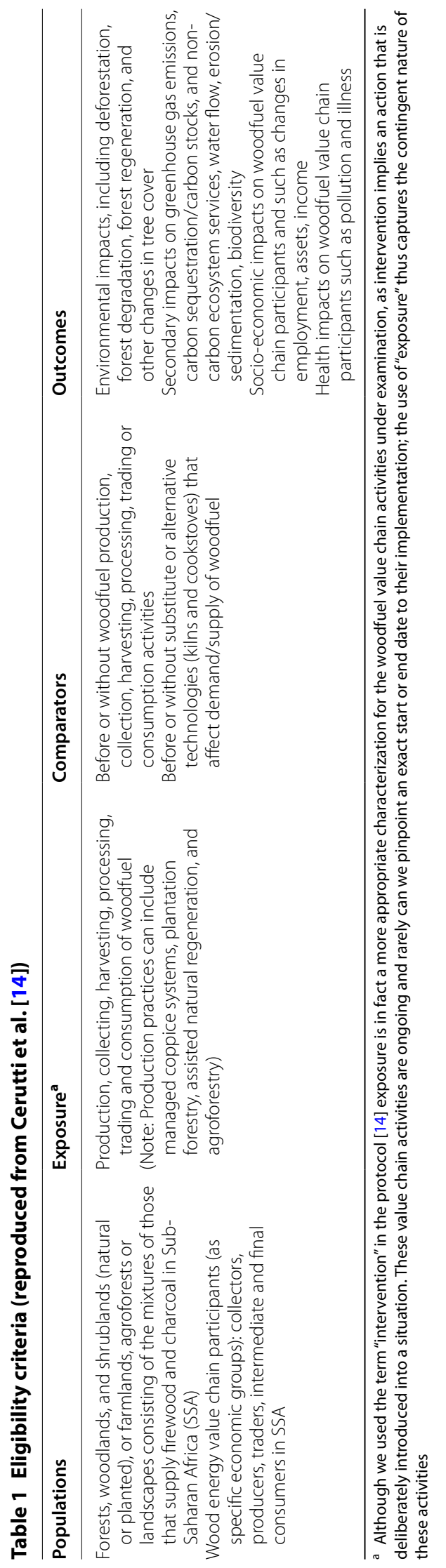


MI, giving a kappa statistic $>0.6$. The five reviewers then continued to complete full text screening using the same eligibility criteria as in the abstract screening (Table 1). The decision rule was to include a study if it met the eligibility criteria for relevant populations, interventions, comparators, and outcomes condition; although comparators were less emphasised at this point.

\section{Study quality assessment}

Full text studies that met the eligibility criteria for inclusion in the review were assessed according to the criteria in Table 2 below. These criteria were expanded from those published in the protocol because most studies did not report sufficient evidence of causation between the data presented and the results and conclusions drawn. The assessment of study methodology was also further subdivided into two questions, as it proved difficult to provide a single appraisal of the multiple criteria used. For each criterion, answers of "yes," "partially," or "no" could be selected from dropdown boxes in an Excel spreadsheet (see Additional file 1: Annexure 2). Studies that failed to meet a minimum assessment were excluded from data extraction. Reviewers individually appraised studies, with the advisory guideline that at least four of the six criteria should be met. Whenever there was doubt on the inclusion of a particular study, reviewers consulted with each other in order to make a final decision.

\section{Study coding strategy}

Using the information in Table 3 from the protocol [14] a template was developed in MSExcel to code study metadata, outcomes, and contextual information. The major categories for coding included the nature of the evidence, representativeness and coverage of evidence, measures of changes/impacts, and other contextual factors, particularly regulatory frameworks for woodfuel. These categories and their respective sub-categories are presented in Table 3. Before individual coding of relevant studies began, reviewers conducted multiple rounds of pilot coding among the entire review team to ensure that there was high agreement on the application of the coding strategy; each round had extensive discussion of discrepancies between reviewers' coding followed by common agreement on a resolution.

\section{Study mapping and presentation}

In presenting the map, we provide first a narrative overview of included articles and their outcomes as they relate to the target population. Further descriptive statistics on quantity, type, focus, study location, and target population of reviewed articles are provided in tables, charts, and graphs.

\section{Results}

\section{Study screening}

A total of 4728 results were retrieved across the three databases of Web of Science, Scopus and CAB Abstracts, which was then reduced to 3979 entries following duplicate removal on Endnote. Title-abstract screening resulted in the identification of 612 articles for full text screening. The review team recommended an additional 21 increasing the number to 633 . However, we were unable to find full texts for 100 of these articles due to limited access to subscription journals and archival materials unavailable through library collections. Following full text screening of the remaining 533 articles by five reviewers (PS, POC, MI, VD, and WZ), a total of 376 articles were excluded as they did not meet the inclusion criteria (Additional file 1: Annexure 2). Most of the studies did not focus on the impacts of woodfuel value chains, (ii) lacked primary data though strong inferences about woodfuel impact were made and (iii) were simply reviews with no primary data. Thus 157 articles were identified for quality assessment.

Table 2 Study quality assessment criteria

\begin{tabular}{ll}
\hline Study components & Criteria \\
\hline $\begin{array}{l}\text { Methodology } \\
\text { Are methods clearly explained and replicable? } \\
\text { Is the sampling frequency, duration of study, and sample size (e.g. extrapolations, generalizations) appropriate for answering the } \\
\text { question(s) posed by the study? } \\
\text { Istudy site selection choice of study site selection clear and justified? This decision will be based on the explanations provided by study authors } \\
\text { regarding a study site's relevance in answering research questions, and is particularly important for the selection of case and } \\
\text { control sites in terms of their comparability } \\
\text { Are the sources of data reliable, complete and available in the article? Do authors acknowledge potential biases, consider } \\
\text { potential confounding factors, and/or perform triangulation to ascertain research results? The reliability of data will be assessed } \\
\text { based on the authors'acknowledgement of potential biases and if triangulation is performed to ascertain research results } \\
\text { Are the results clearly linked to the intervention? Is causation of the socioeconomic, environmental, and/or health outcomes } \\
\text { clearly attributable to the wood energy activities? } \\
\text { Are the conclusions justified by the results presented? Are the authors' conclusions about the impact of woodfuel value chain } \\
\text { activities supported by their data? }\end{array}$ \\
$\begin{array}{l}\text { Results } \\
\text { Conclusion }\end{array}$
\end{tabular}


Table 3 Study coding categories

\begin{tabular}{|c|c|}
\hline Nature of evidence & $\begin{array}{l}\text { Sources of evidence (journal types and subjects, grey literature), Type of study (socio economic, } \\
\text { environmental) }\end{array}$ \\
\hline Representativeness and coverage of evidence & $\begin{array}{l}\text { Geographic coverage (scope, location, scale) } \\
\text { Focus (firewood, charcoal, other related energy sources) } \\
\text { Populations (value chain participants, Forest type, Information on agro ecological zone, Tenure and } \\
\text { Sample size and of population of interest) }\end{array}$ \\
\hline Measure of changes/impacts & $\begin{array}{l}\text { Nature of outcomes reported (increase, decrease, no change/neutral) } \\
\text { Socio-economic (income, employment, asset, equity, costs, profit) } \\
\text { Environmental (deforestation, forest area, degradation, biodiversity, C stocks regeneration, ecosystem } \\
\text { services) } \\
\text { List of outcomes that are not comparative in nature, but relevant to answer the review questions }\end{array}$ \\
\hline Context & Regulatory framework described (trade, energy, environment) \\
\hline
\end{tabular}

\section{Quality assessment}

Following study quality assessment of 157 articles, 26 articles were removed for failing to meet the minimum set criteria. Studies were excluded at this stage if they failed on four of the six criterion (Additional file 2: Annexure 3). The major weaknesses in the studies were poor study designs rendering data unreliable to support presented results and conclusions.

\section{Study coding}

The remaining 131 articles entered study coding stage. Due to the presence of multiple populations, interventions, or comparators in some articles that may have also resulted in correspondingly different impacts, the final study coding had a total of 152 individual studies. The systematic map database is presented in the MS Excel spreadsheet (Additional file 3: Annexure 4) and organized into five main categories: (i) general information about each study, including geographic scope and research methods; (ii) type and baseline condition of the ecosystem where woodfuel extraction is taking place; (iii) the specific woodfuel activities assessed, as well as the introduction (if any) of woodfuel policies or alternative technologies; (iv) contextual factors, including tenure regime characteristics, population dynamics, socioeconomic condition, and market demand, and (v) study outcomes as they relate to predetermined environmental, socioeconomic, and health indicators. Additional file 4: Annexure 5 presents full references for this map.

\section{Article publication trends}

Over the last decade, there has been a steady rise in the number of articles published on the impacts of woodfuel value chains in SSA (Fig. 2). Of the 131 articles eventually included in this systematic map, $80 \%$ were published in 2000 or later, with articles published in or after 2010 accounting for $44 \%$ of the total evidence base.

Of the 152 studies, 93 examined environmental impacts, 60 socio-economic impacts, and 27 health impacts. Studies in the systematic map came from 26 of the 49 Sub-Saharan Africa countries (Fig. 3), with a near even distribution across southern, eastern and western African countries with 50, 46 and 44 of the 152 studies respectively. Tanzania, South Africa, and Nigeria were the most represented countries, with 21,18 , and 17 studies, respectively, while all other countries had ten or fewer.

The majority of studies were conducted at sub national level or lower, with $66 \%$ conducted at the village or city scale or smaller. For instance, 36\% (55 studies) were conducted at village or city levels, 19\% (29 studies) in sample plots and 11\% (17 studies) at designated areas or reserves. This phenomenon was more common with the socio economic studies where $92 \%$ of studies were conducted on sub-national levels (provincial or local village level). Equally, health studies were dominated by village/ city (50\%) level data whilst environmental studies were mainly (72\%) conducted on plots, small designated areas or at village/city scales (Fig. 4).

\section{Methods used by the studies}

Various comparative methods were used, although case studies (56\%) were the most dominate. Comparators were used by 33, 19 and $37 \%$ of the environmental, socioeconomic and health studies respectively. In the 66 studies with comparators authors most often used control-impact designs (34 studies) to compare impacts between the intervention site with another where the intervention was not present (Table 4).

\section{Woodfuel interventions and contextual factors}

Of the 152 studies, 77 (51\%) reported on more than one woodfuel value chain activity. Harvesting and consumption were the most frequently reported activities in $88(58 \%)$ and $67(44 \%)$ studies respectively, whilst tree growing and management of natural trees were least frequently studied (Fig. 5).

Few studies explicitly studied the contribution or impacts of policy reform on woodfuel value chains: 52 studies (34\%) presented data on woodfuel policy changes 


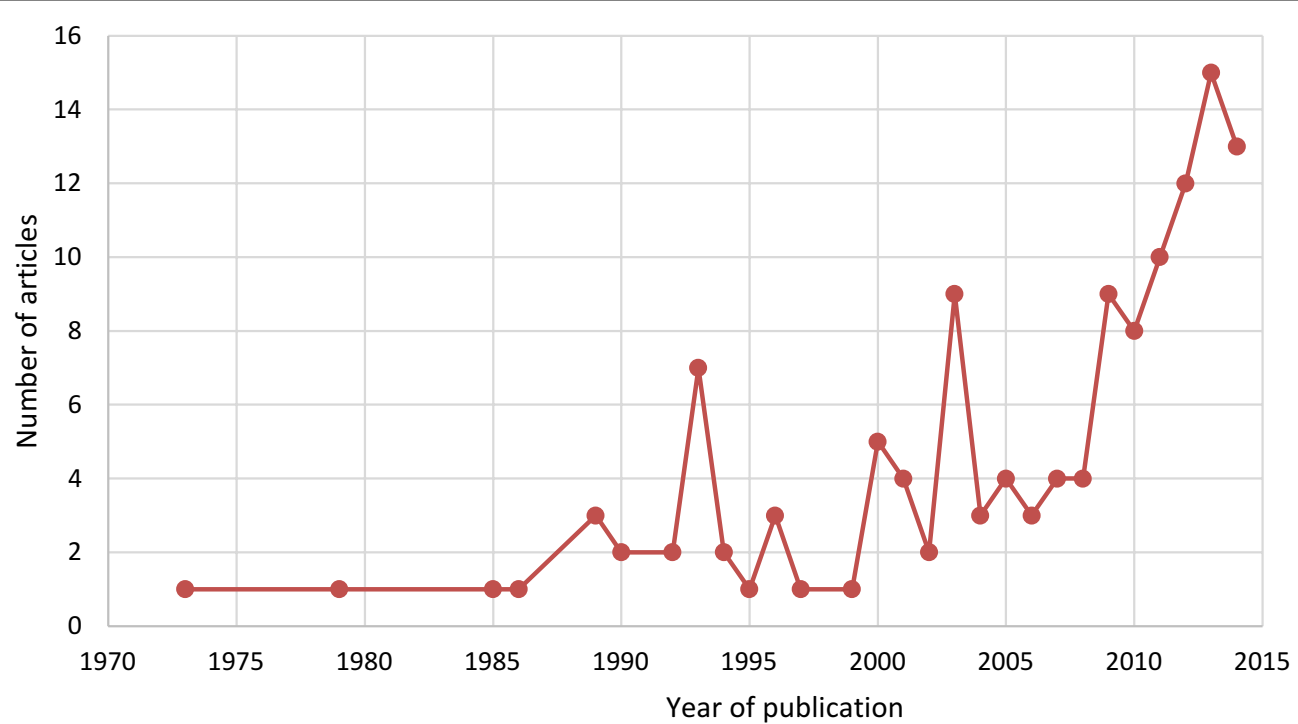

Fig. 2 Articles by year of publication

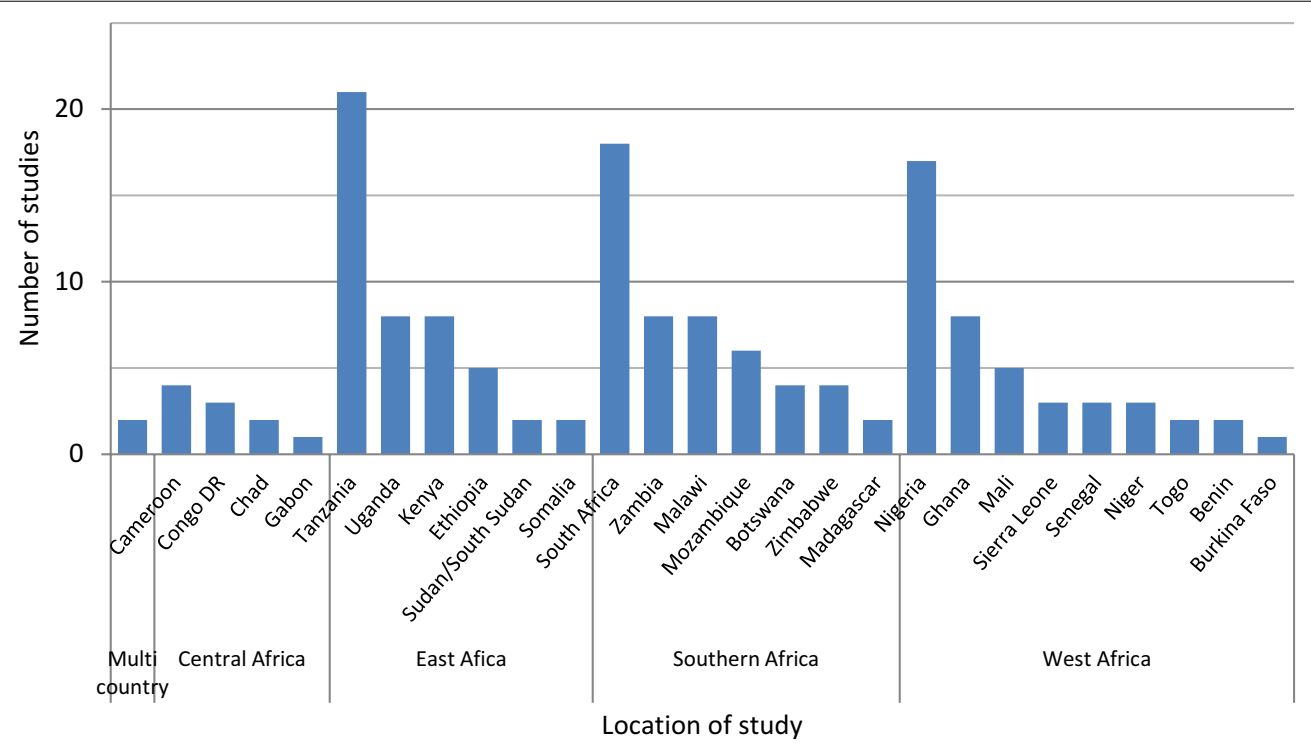

Fig. 3 Number of studies per country

that were implemented at the study areas. Very few studies (5 studies) were conducted after 15 years of policy implementation. Of the 52 studies, nearly half ( 25 studies) had multiple policy targets within the woodfuel value chain activities. Trade/marketing (30\%) and harvesting (33\%) were the most targeted activities. In addition, 23 studies (15\%) examined the introduction of alternative technologies for consumption reduction such as wood saving stoves. Only eight studies (5\%) made the link between alternative technologies and their scaling-up through policy changes that would lessen demand on natural resources.
Sixty-four percent (97 studies) of studies reported additional contextual factors that influenced the direction of environmental, socioeconomic, and health outcomes that were not included in the main data extraction (Fig. 6). The most frequently reported factor was agricultural expansion (31 studies) which was linked to deforestation as well as serving as a new source of woodfuel. Fire, livestock grazing, and woodcutting were reported as much as large scale factors like urbanization, and infrastructure development, as well as enabling factors such as governance, property rights, unemployment and gender. 


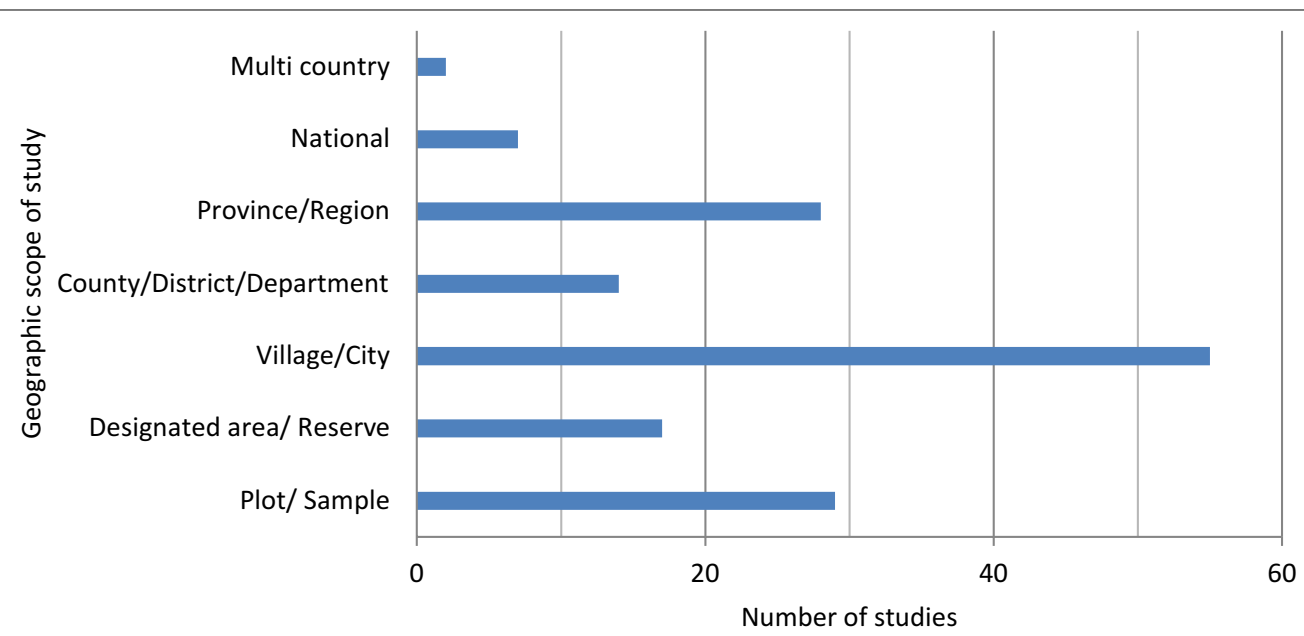

Fig. 4 Scope of the studies

Table 4 Distribution of studies by outcome area and comparators used

\begin{tabular}{|c|c|c|c|c|c|c|c|c|}
\hline \multirow{2}{*}{ Comparator } & \multicolumn{2}{|c|}{ Total } & \multicolumn{2}{|c|}{ Environmental studies } & \multicolumn{2}{|c|}{ Socio economic studies } & \multicolumn{2}{|c|}{ Health studies } \\
\hline & No. & $\%$ & No. & $\%$ & No. & $\%$ & No. & $\%$ \\
\hline BACI (before-after and control-impact) & 4 & 3 & 3 & 3 & 4 & 7 & 0 & 0 \\
\hline Before-after & 3 & 2 & 0 & 0 & 0 & 0 & 0 & 0 \\
\hline Control-impact & 34 & 22 & 26 & 28 & 3 & 5 & 7 & 26 \\
\hline Counterfactual & 7 & 5 & 2 & 2 & 4 & 7 & 3 & 11 \\
\hline Multiple cases & 25 & 16 & 10 & 11 & 16 & 27 & 1 & 4 \\
\hline Single case & 61 & 40 & 38 & 41 & 30 & 50 & 13 & 48 \\
\hline Time series & 18 & 12 & 14 & 15 & 3 & 5 & 3 & 11 \\
\hline Total & 152 & & 93 & & 60 & & 27 & \\
\hline
\end{tabular}

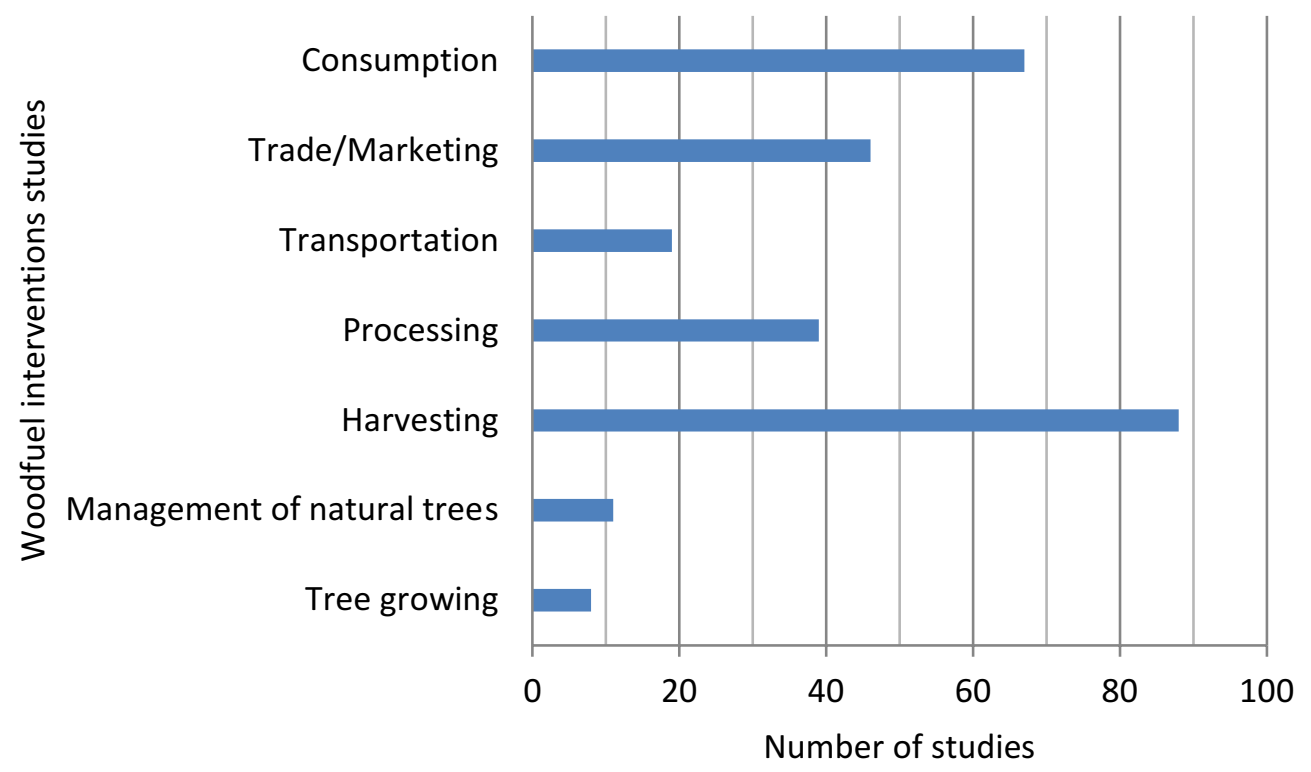

Fig. 5 Woodfuel value chain interventions studied 


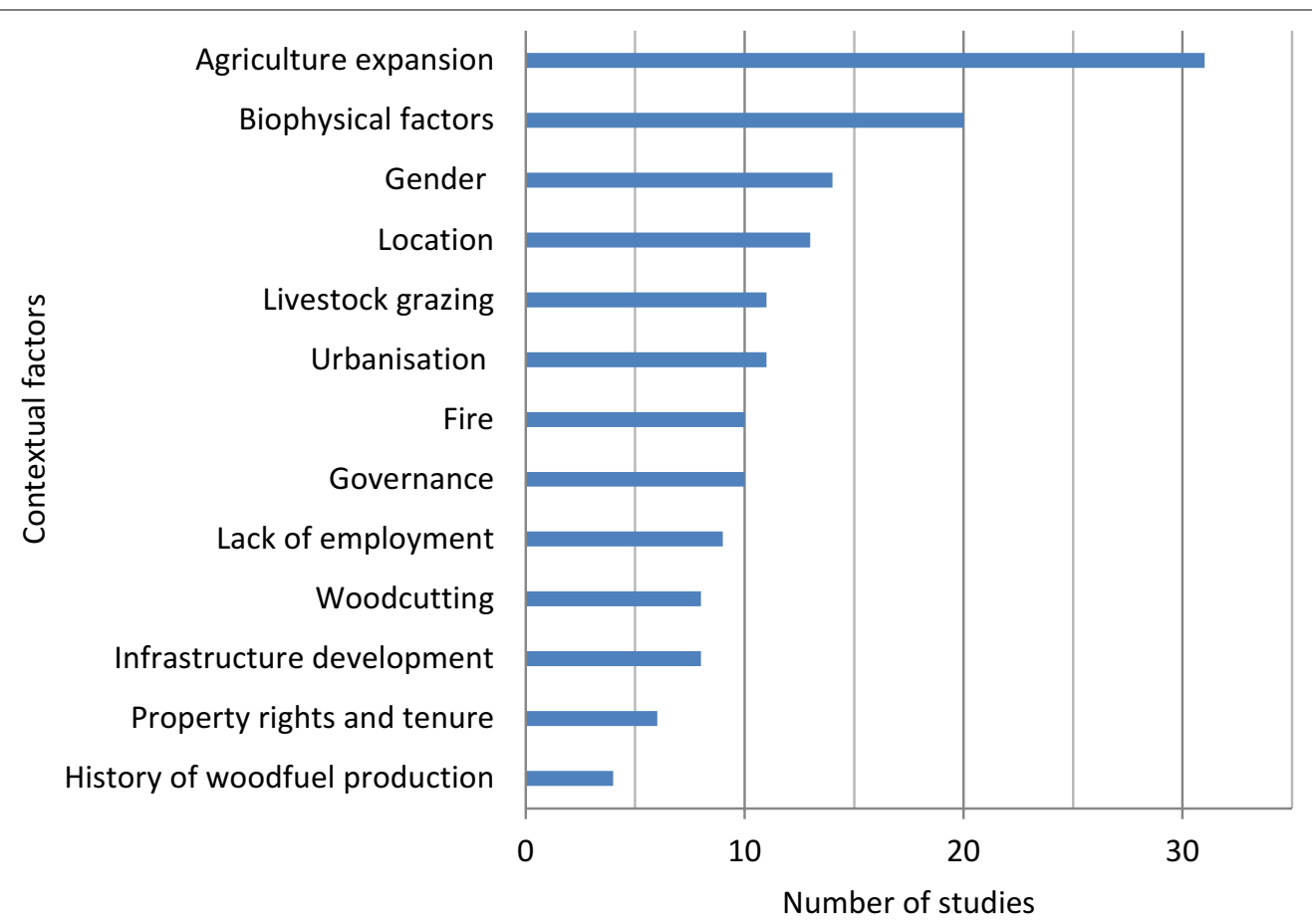

Fig. 6 Other contextual factors reported

Sixty-eight studies (45\%) reported on the tenure regimes existing in the study sites. Thirty-four studies (22\%) reported mixed tenure regimes, followed by studies located on state land (12\%), community ownership (9\%) and lastly, private ownership (5\%). Furthermore, 20 studies reported the influence of biophysical factors, such as presence of drought or pests, wet/dry season climatic differences, and geological or vegetation characteristics that may affect rates of regeneration.

\section{Baselines and impacts}

\section{Environmental and socioeconomic baselines}

The terms "woodlands" and "savanna" were often used interchangeably by studies, and so the two categories here may be understood to refer to the same biome; the same applies to trees on farm and agro-forests. Mixed biomes represents mosaics of woodlands forests, shrubs, and or grasslands. Most studies (110 studies) reported the forest type of the study area; most studies were conducted in savannas/woodlands (53\%) and forests (26\%) (Fig. 7).

Furthermore, most of the studies were conducted in primarily natural vegetation (54\%), followed by those conducted on both planted and natural vegetation $(12 \%)$ and on plantations only (3\%). The remaining studies (46\%) did not specify whether the vegetation was natural or planted. Fifty-four studies (36\%) reported baseline environmental conditions of the ecosystem prior to the start of woodfuel interventions. Of those, only three studies (3\%) reported good or excellent condition at study sites.

Sixty seven studies (44\%) focused on domestic consumption of woodfuel, 45 studies (30\%) on its commercial use and 38 studies (25\%) reported on both. Furthermore, forty-four studies (29\%) reported on the distance to the nearest market or urban centre: 24 studies (54\%) were located within $10 \mathrm{~km}$ of a market, while ten studies (23\%) were located $60 \mathrm{~km}$ or further. However, very few studies (8\%) provided information on population dynamics at the study site.

\section{Environmental impacts}

Studies that reported environmental outcomes came from a limited geographic scope, with $53 \%$ of studies located in only five countries; namely South Africa, Tanzania, Zambia, Nigeria and Kenya (Fig. 8). Firewood was studied more frequently than charcoal (51 versus $32 \%$, respectively), although $17 \%$ of the studies examined both forms of woodfuel. In addition, the type of fuel studied in evaluating environmental outcomes varied across the countries. For instance, all 15 studies from South Africa studied solely firewood, while studies from Kenya, Zambia and Tanzania focused more on charcoal.

Forty-four (29\%) studies were conducted in natural forests, while $12(8 \%)$ were conducted in both natural and 


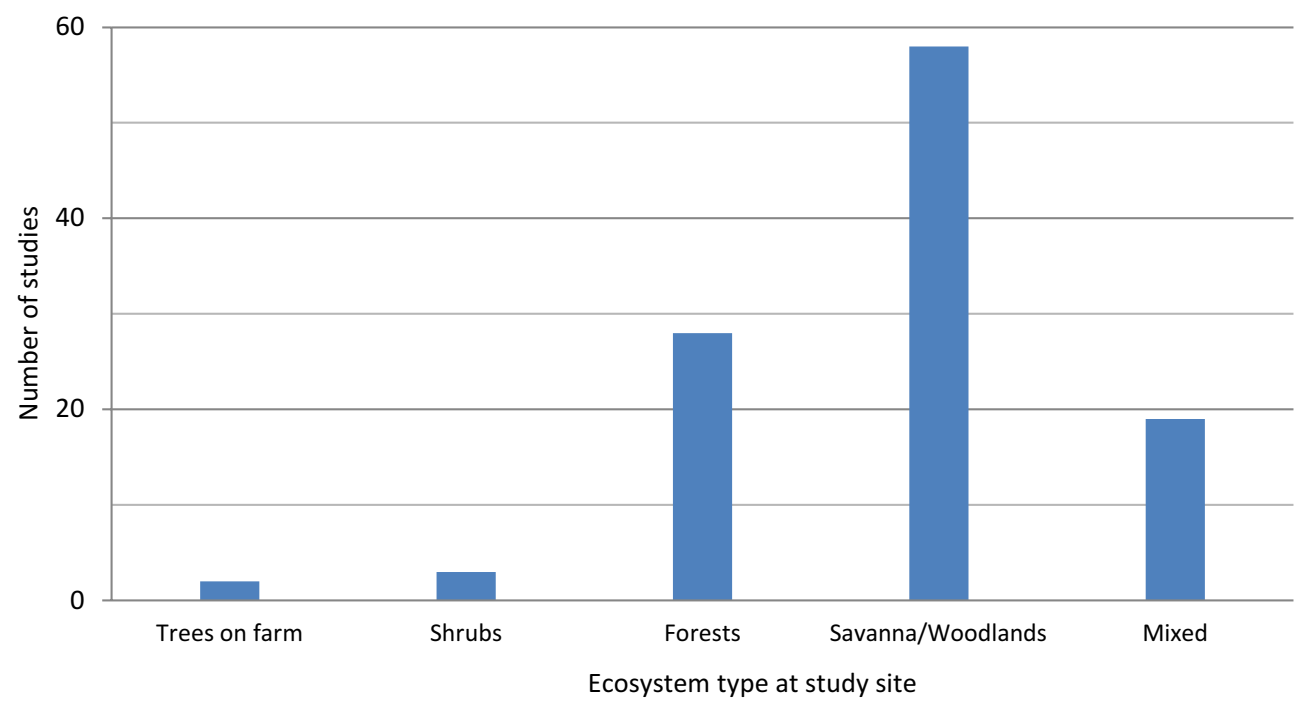

Fig. 7 Types of ecosystems reported in 110 studies

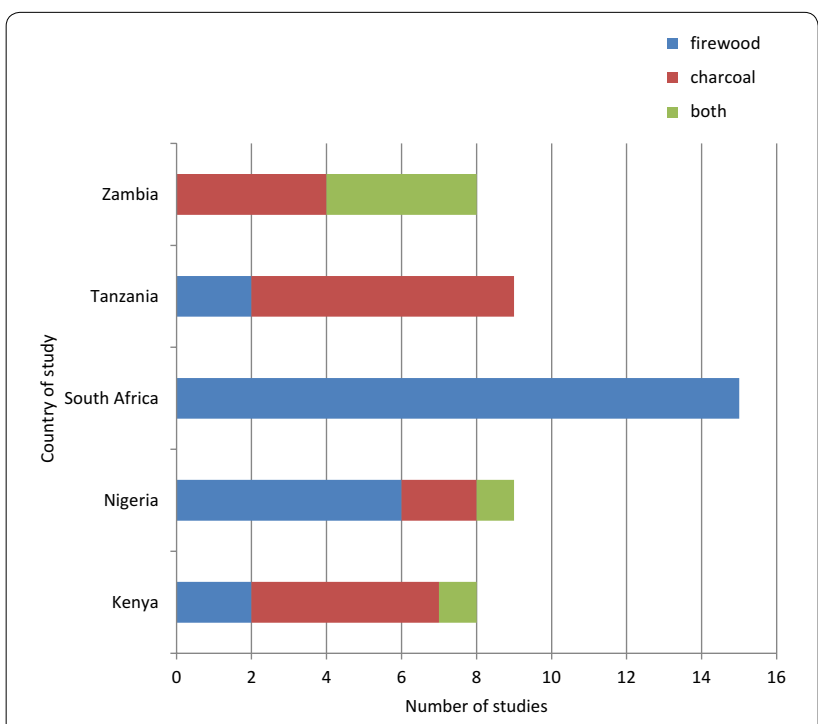

Fig. 8 Woodfuel studied in assessing environmental impacts

planted forests. Changes in forest cover was the most frequently reported outcome (69\%), followed by changes in forest condition in 47 studies (51\%) and biodiversity in 28 studies (30\%) (Table 5). However, there was a lot of variation in terms of the direction of change a (positive, negative or none) among the studies as detailed in Additional file 3: Annexure 4. Twenty seven studies reported linkages between use and environmental conditions under commercial and domestic and use of living trees versus deadwood (Fig. 9).

\section{Socio-economic impacts}

A total of 60 studies addressed socio-economic impacts, with a nearly equal division of studies that examined charcoal (35\%), firewood (31\%) or both sources of energy (34\%). The studies were undertaken in 20 Sub-Saharan African countries with a majority from East Africa, notably Tanzania (10 studies) and Uganda (5 studies); all other countries had one to three studies each. Changes in incomes and profits were the main socio-economic impacts reported for value chain actors, although there were considerable proportions of studies reporting on equity labour; gender aspects; community funds and conflicts (Fig. 10).

\section{Environmental and socio-economic trade-offs}

Twenty nine studies (19\%) presented both socioeconomic and environmental impacts. Most of them found trade-offs between environmental and socioeconomic outcomes. Only one study presented study presented environmental and health outcomes and none was on health and socio-economic outcomes.

\section{Health impacts}

Twenty seven studies, 27 (18\%) presented empirical evidence of the impact of woodfuel consumption on household health. Studies on health impacts came from only 12 of the 49 SSA countries most of which were from West Africa (50\%), of which Nigeria constituted 29\% of regional studies, followed by Southern Africa (29\%), whereas no studies were reported from Central Africa (Table 6). Studies reported on both types of woodfuel, 
Table 5 Environmental impact indicators

\begin{tabular}{|c|c|c|c|c|c|c|}
\hline Impact indicator & $\begin{array}{l}\text { Forest cover (natural or } \\
\text { planted) }\end{array}$ & $\begin{array}{l}\text { Forest condition, } \\
\text { including forest structure }\end{array}$ & Biodiversity & Carbon stocks & GHG emissions & Soil quality \\
\hline Total number of studies & 64 & 47 & 28 & 15 & 5 & 16 \\
\hline
\end{tabular}

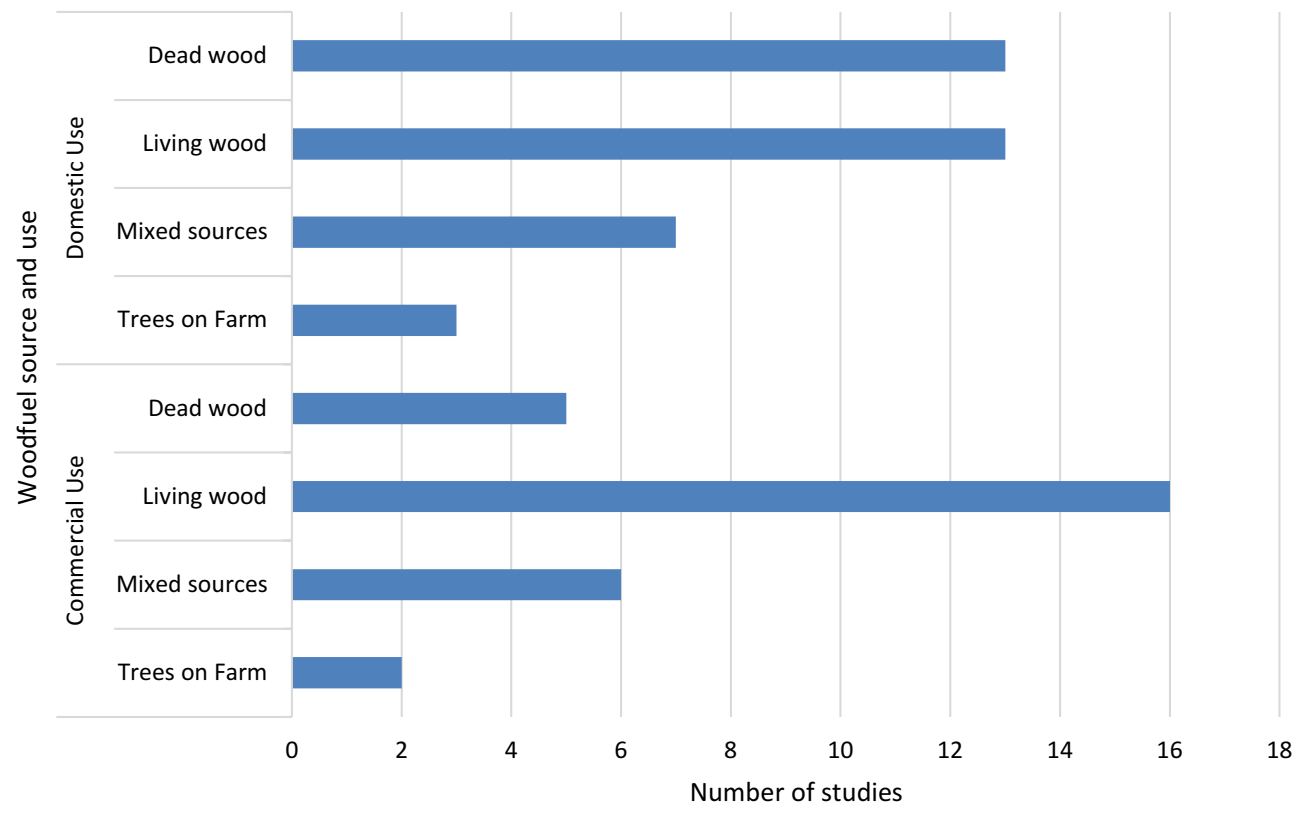

Fig. 9 Primary source and use of woodfuel

firewood, charcoal. However, more of the health studies (74\%) assessed domestic consumption than commercial use. Impacts recorded were mainly related to illness (19 studies) (acute respiratory infection, chronic bronchitis, impaired lung function etc.) and pollution (16 studies). Other impacts included birth defects such as low birth weight and low foetal growth, as well as presence of pollutants that include gaseous emissions and solid particulates. Some of the main factors contributing to these health impacts as presented in the studies include prevalence of indoor cooking, poor ventilation in kitchens, low education levels and low social status of women.

\section{Discussion}

There has been great interest in woodfuel issues in SSA since the late 1970s. The search capacities of this exercise have found a particular preponderance of articles published in the last ten years, (as shown by the number of articles retrieved and screened for this systematic map). However if the time and resources to go back to the nondigitized earlier grey literature had been available, many more would have been unearthed from that period. Most articles $(40 \%)$ did not meet the minimum standard chosen for this map during critical appraisal. An exception is represented by studies reporting health impacts, most of which were relatively better designed, e.g. by incorporating comparators. However, only 4 studies (3\%) in total used BACI designs.

The sudden increase in the number of articles published after 2000 may also be due to renewed research and policy interest in the topic following disappointment over the failures of woodfuel interventions in previous decades [18]. Another factor possibly contributing to more literature being published could be related to the increasing number of perspectives on the paradigm of a transition from woodfuels to modern energy sources as being necessary to raise SSA countries out of poverty and onto developmental pathways $[19,20]$.

Findings suggest that woodfuel value chains have environmental, socio economic and health consequences with the frequent presence of trade-offs. Yet, as said, most studies did not meet the methodological standards required by the critical appraisal, hence such reported impacts were not measured against baselines or comparators, making attribution of impacts to woodfuel activities and policy interventions, very difficult to substantiate. This results in a knowledge gap that we argue should be addressed in future research. For instance, in the case of 


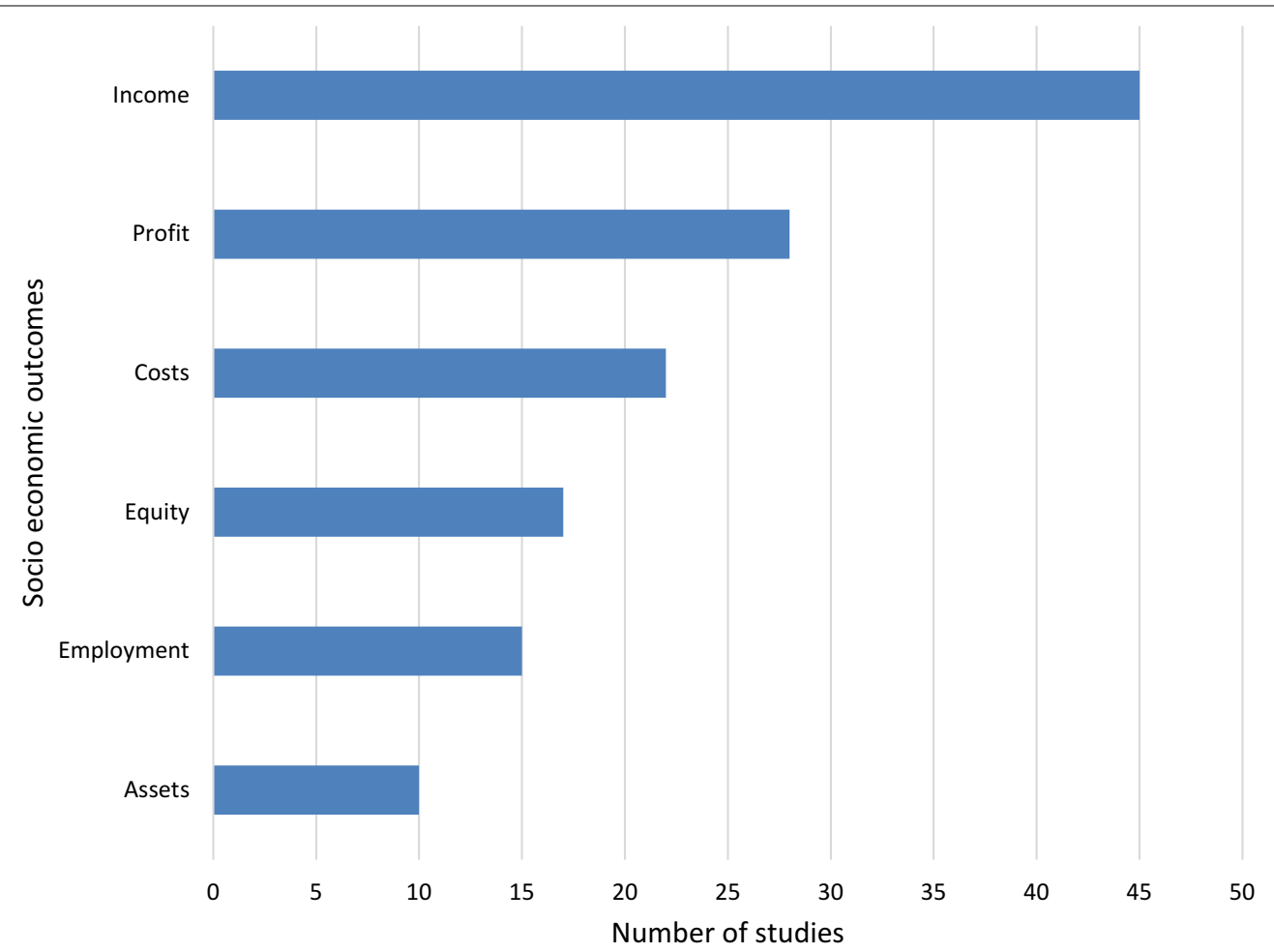

Fig. 10 Socio-economic outcome indicators (multiple counts from 51 studies)

Table 6 Countries from which data on health impacts was generated

\begin{tabular}{llrr}
\hline Region & Countries & Number of countries & Number of studies \\
\hline East Africa & Ethiopia, Kenya, Tanzania & 3 & 5 \\
Southern Africa & Malawi, Mozambique, South Africa, Zimbabwe & 4 & 8 \\
West Africa & Burkina Faso, Ghana, Nigeria, Senegal, Sierra Leone & 5 & 14 \\
SSA & & 12 & 1 \\
Total & & 12 & 28 \\
\hline
\end{tabular}

socio-economic studies, the lack of comparators made it difficult to determine whether woodfuel value chain participants saw improved livelihoods relative to non-participants. As such lack of comparison to relevant control groups and other income generating activities means that we cannot definitively attribute overall livelihood gains (or losses) to woodfuel value chain activities.

Similarly, studies reporting environmental outcomes provided limited evidence that would make attribution to wood energy activities possible. For example, studies that employed satellite images to track land use change or compare different sites often failed to give concrete evidence differentiating the impacts of woodfuel value chain activities from other land use changes that may instead have been the primary causes of reported deforestation or forest degradation. Therefore, further research should take into consideration the influence of key contextual factors such as agricultural expansion, urbanization, governance, and tenure regimes, as these may well have greater causal effect on forest degradation and deforestation than the proximate activities of woodfuel harvesting, trade, and consumption.

Use of woodfuel for domestic cooking is reported to result in indoor pollution and subsequently health problems. However, additional factors that could contribute to these two impacts, such as the location of the cooking site, design/ventilation of kitchens and general social and economic status of the users under study, and thereby their access to alternative (and cleaner) forms of energy should be of particular focus in future studies.

Besides inadequate baseline information and lack of comparators, a knowledge gap also arises from the studies' limited geographic scope. Moreover, the studies do not necessarily represent outcomes at national levels, as almost all of them were conducted on either provincial or 
village scales, focusing on locations where woodfuel production and trade are of local importance. This in itself could potentially introduce a bias resulting in areas of low woodfuel use being neglected by research.

The predominance of small-scale studies makes generalization of the map results difficult as they may be very specific to the particular study sites and countries where they were conducted. We are aware that generalizations are not always necessary or needed, but the particular fragmentation found by this map is a matter of concern if national or regional policies must be adopted by policy makers, as the representativeness of the findings for wider application is certainly limited.

Lastly, from the literature assessed, it is also very difficult to map the impacts of woodfuel demand separately from supply, as most studies do not explicitly consider this key differentiation in their methodologies, or the trade-offs that might exist among them. This is relevant because adopting policies that specifically target only one part of the value chain and its operators might result in counterintuitive (and possibly negative) impacts on other interrelated parts of the value chain. For example, studies discussing environmental impacts focused more on woodfuel supply; studies on health outcomes focused mostly on consumption, whilst socioeconomic studies covered a wider range of woodfuel value chain activities except for consumption. Although methodologically more challenging, limited by funding and time constraints, we argue future research should aim for improved overall assessments of the entire value chain.

\section{Limitations of the map}

The search did not include the additional specialist websites and internet search engines defined in our protocol due to time constraints. This led to inclusion of fewer grey literature references than might have otherwise been the case, and consequently fewer references to the literature of the 1980 and 1990s which was produced before electronic archiving of journal articles. In addition, the search strategy could not overcome the inherent problem of English language bias which has been termed the 'Tower of Babel' bias [21, 22]; where most authors choose to publish significant results in English rather than other languages. There is also the potential influence of publication bias, wherein significant results are published with far greater likelihood than null results. Therefore, more work is required in consolidating evidence from the grey literature and other languages in future syntheses on this subject matter.

\section{Conclusions}

\section{State of the evidence base}

From this systematic map we conclude that there is inadequate evidence on the outcomes of woodfuel value chains in SSA. Although many studies have been written on the subject, most articles did not meet the minimum standard chosen for this map during quality assessment falling short on the methodologies and representativeness making attribution very difficult especially for environmental and social impact studies. For instance only a handful used comparators and or considered contextual factors that could confound the results. In addition the studies were from a very limited geographic scope with only 26 of the 49 countries represented. Furthermore studies on environmental impacts were from only five countries. Overall, failure to meet our minimum standard speaks more to the very limited potential of the existing literature to inform broad policies and generalizations across SSA. Thus further simplification of the research questions might not yield any better results as the evidence base is very weak.

\section{Implications for policy and management}

This systematic map suggests that there are environmental, socioeconomic and health consequences associated with woodfuel value chains in Sub Saharan Africa. However, the literature also shows a weak and geographically limited evidence base to justify the above claims. Notwithstanding the well-known disconnect between science and policy even under the best conditions, we argue that policy formulation processes targeting woodfuels in SSA deserve a more solid, coherent and broad body of knowledge, especially on such a vital sector of rural economies.

\section{Implications for research}

There is an urgent need to design and undertake research using robust methodologies at appropriate scales in order to make substantial conclusions and policy recommendations about the outcomes and impacts of woodfuel value chains in SSA. Research designs with appropriate comparators is particularly critical, in order to ensure methodological rigor and reliability of results. Equally important are long-term studies with corresponding baseline information of initial socioeconomic and environmental conditions. In addition very few studies managed to assess trade-offs or provide quality analysis on linkages between socio-economic, environmental and health outcomes, often focusing on one aspect and neglecting potential impacts on the other. Although it is unrealistic to expect every study to assess a broad set of impacts-especially considering that health studies often come from different concerns than environmental ones-we believe that a more robust research that takes into account the interrelationships between environmental and socio-economic outcomes, deserve more attention, if substantial conclusions are to be drawn and policies better. For instance, environmental conditions (e.g. type of the ecosystem, type and sources of woodfuel) could have a bearing on the ultimate 
socio-economic and health outcomes as much as contextual factors (e.g. tenure, technology use, markets, policies and population dynamics) could influence the extent and direction of environmental and socio-economic impacts. Further studies should also focus more in countries under-represented in the literature.

\section{Additional files}

Additional file 1: Annexure 2. Woodfuel systematic map full text screening exclusions.

Additional file 2: Annexure 3. Woodfuel systematic map quality assessment.

Additional file 3: Annexure 4. Woodfuel systematic map.

Additional file 4: Annexure 5. Woodfuel systematic map references.

\section{Abbreviations}

CGIAR: Consultative Group of International Agricultural Research; CIFOR: Center of International Forestry Research; GHG: green house gases; ICRAF: World Agroforestry Centre; IEA: International Energy Agency; KNOW-FOR: International Forestry Knowledge; LPG: liquid petroleum gas; MS Excel: microsoft excel; SSA: Sub-Saharan Africa; UK: United Kingdom; WHO: World Health Organisation; WOS: Web of Science.

\section{Authors' contributions}

PS, POC, WZ, MI, AC, JY, DG and JS collaborated in the screening and data extraction for the map as well as drafting the initial manuscript, with writing led by PS and POC. The final draft of the manuscript received substantial inputs from all authors. All authors read and approved the final manuscript.

\begin{abstract}
Author details
${ }^{1}$ World Agroforestry Centre (ICRAF), United Nations Avenue, Gigiri, PO Box 30677, Nairobi 00100, Kenya. ${ }^{2}$ Center for International Forestry Research (CIFOR), Jalan CIFOR, Situ Gede, Sindang Barang, Bogor (Barat) 16115, Indonesia. ${ }^{3}$ Agricultural Research Centre for International Development (CIRAD), Avenue Agropolis, 34398 Montpellier Cedex 5, France. ${ }^{4}$ Japan International Research Center for Agricultural Sciences (JIRCAS), 1-1 Ohwashi, Tsukuba, Ibaraki 305-8666, Japan. ${ }^{5}$ Forest and Nature Conservation Policy Group, Schure Research, Wageningen UR, PO Box 47, Wageningen, The Netherlands. ${ }^{6}$ African Forest Forum (AFF), United Nations Avenue, Gigiri, P.O. Box 30677 , Nairobi 00100 , Kenya. ${ }^{7}$ Department of Environmental Studies, Kenyatta University, Nairobi, Kenya. ${ }^{8}$ Department of Zoology, University of Oxford, South Parks Road, Oxford, UK. ${ }^{9}$ Department of International Development, London School of Economics, 6-8th Floors, Connaught House, London School of Economics and Political Science, Houghton Street, London WC2A 2AE, England, UK.
\end{abstract}

\section{Competing interests}

There are no known competing interests. During screening and quality assessment, articles that were authored by members of the review team were assigned to other reviewers to ensure that decision-making would not be unduly biased.

\section{Funding}

This work has been conducted with the financial assistance of the UK Department for International Development, through its KNOW-FOR program grant to the Center for International Forestry Research (CIFOR), and funding partners who have contributed to the CGIAR Fund.

\section{Annex 1 Search string}

Web of Science, 19 January 2015 (UMich access)

TOPIC: (Forest* OR wood* OR shrub* OR bush* OR producer* OR trader* OR middle*men OR market* OR seller* OR vendor* OR retailer* OR wholesale* OR transport" OR buyer* OR consumer* OR household* OR communit* OR smallholder*)

AND

TOPIC: ("wood fuel" OR "wood energy" OR "fuel energy" OR "fuel use" OR firewood OR fuel"wood OR deadwood OR charcoal OR biofuel OR "biomass fuel" OR "biomass energy")

AND

TOPIC: (produc* OR harvest* OR process* OR trad* OR market" OR consum* OR supply OR demand OR buy* OR purchas* OR pick* OR collect* OR usage OR use OR utilize $\left.{ }^{*}\right)$

AND

TOPIC: (Angola* OR Benin OR Botswana OR "Burkina Faso" OR Burundi OR Cameroon" OR "Cape Verde" OR "Central African Republic" OR Chad OR Comoros OR Congo OR "Cote d'Ivoire" OR "Ivory Coast" OR Djibouti OR "Equatorial Guinea" OR Eritrea* OR Ethiopia* OR Gabon* OR Gambia* OR Ghana* OR Guinea* OR "Guinea-Bissau" OR Kenya* OR Lesotho OR Liberia* OR Madagascar OR Malawi" OR Mali* OR Mauritania* OR Mauriti* OR Mozambiq* OR Namibia* OR Niger* OR Reunion OR Rwanda* OR "Sao Tome and Príncipe" OR Senegal" OR Seychelles OR "Sierra Leone" OR Somalia" OR "South Africa" OR Sudan* OR Swaziland OR Tanzania* OR Togo OR Uganda* OR Zambia* OR Zimbabwe* OR sub"saharan or West"Africa or East"Africa or "southern Africa" or "central Africa" or "horn of Africa")1595 results $\rightarrow 1579$ results after duplicate removal.

\section{Scopus, 19 January 2015}

TITLE-ABS-KEY (forest* OR wood* OR shrub* OR bush" OR producer OR trader OR middle*men OR market* OR seller OR vendor OR retailer OR wholesale* OR transport* OR buyer OR consumer OR household OR communit* OR smallholder)

AND

TITLE-ABS-KEY (\{wood fuel\} OR \{wood energy\} OR \{fuel energy\} OR \{fuel use\} OR firewood OR fuel"wood OR deadwood OR charcoal OR biofuel* OR "biomass fuel" OR "biomass energy")

AND

TITLE-ABS-KEY ((produc* OR harvest* OR pro-

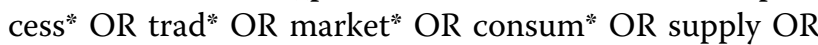
demand OR buy* OR purchas* OR pick* OR collect* OR usage OR use OR utilize*) AND (angola* OR benin OR botswana OR \{Burkina Faso\} OR burundi OR cameroon* OR \{Cape Verde\} OR \{Central African Republic\} OR chad OR comoros OR congo OR \{Cote d'Ivoire\} OR \{Ivory Coast\} OR djibouti OR \{Equatorial Guinea\} OR eritrea* OR ethiopia* OR gabon* OR gambia* OR ghana* OR guinea* OR \{Guinea-Bissau\} OR kenya* OR lesotho 
OR liberia* OR madagascar OR malawi* OR mali* OR mauritania* OR mauriti* OR mozambiq* OR namibia* OR niger* OR reunion OR rwanda* OR \{Sao Tome and Principe\} OR \{Sao Tome and Príncipe\} OR senegal* OR seychelles OR \{Sierra Leone\} OR somalia* OR \{South Africa\} OR sudan* OR swaziland OR tanzania* OR togo OR uganda* OR zambia* OR zimbabwe* OR sub"saharan OR west"africa OR east"africa OR \{southern Africa\} OR \{central Africa\} OR \{horn of Africa\}) AND (income* OR livelihood* OR health OR gender OR education OR perception* OR attitude* OR deforest* OR degrad* OR reforest" OR regrowth OR "biodiversity conservation" OR "species loss" OR "species decline" OR "species gain" OR "species increase" OR "ecosystem services" OR policy OR legislation OR regulat" OR law OR legal OR manage* OR administration OR customary OR rules OR bylaws OR alternative OR substitute OR kiln OR cookstoves))

AND

EXCLUDE (SUBJAREA, "MEDI") OR EXCLUDE (SUBJAREA, "ENGI") OR EXCLUDE (SUBJAREA, "BIOC") OR EXCLUDE (SUBJAREA, "CENG") OR EXCLUDE (SUBJAREA, "IMMU") OR EXCLUDE (SUBJAREA, "CHEM") OR EXCLUDE (SUBJAREA, "PHAR") OR EXCLUDE (SUBJAREA, "ARTS") OR EXCLUDE (SUBJAREA, "BUSI") OR EXCLUDE (SUBJAREA, "PHYS") OR EXCLUDE (SUBJAREA, "MATE") OR EXCLUDE (SUBJAREA, "COMP") OR EXCLUDE (SUBJAREA, "VETE") OR EXCLUDE (SUBJAREA, "MATH") OR EXCLUDE (SUBJAREA, "DECI") OR EXCLUDE (SUBJAREA, "NURS") OR EXCLUDE (SUBJAREA, "HEAL") OR EXCLUDE (SUBJAREA, "PSYC") OR EXCLUDE (SUBJAREA, "DENT") OR EXCLUDE (SUBJAREA, "NEUR")2104 results $\rightarrow$ only 2000 results can be exported; thus 2000 results organized based on relevance were exported $\rightarrow 1997$ records following duplicate removal.

\section{CAB search (Oxford)}

(communit* or household* or smallholder* or market*). af. OR (forest" or shrub* or bush" or wood*).af. OR (producer* or trader* or seller* or vendor* or retailer* or wholesaler" or transporter" or buyer" or middlemen).af.

AND

(wood\$fuel or "biomass fuel" or "biomass energy" or "wood energy" or "fuel energy").af. OR (fire\$wood or fuel\$wood or deadwood or charcoal or biofuel).af.

AND

(produc* or harvest" or pick" or collect" or utili" or process" $^{*}$ or trad" or market" or consum* or supply or dem and or buy" or us*).af. OR (policy or legislation or law or "legal framework" or "legal provisions" or administration or customary or rules or by\$law*).af.

AND ("southern Africa" or "central Africa" or "horn of Africa"). af. OR ("East Africa or "eastern Africa").af. OR "West Africa" or "western Africa").af. OR (Angola or Benin or Botswana or Burkina Faso or Burundi or Cameroon or Cape Verde or Central African Republic or Chad or Comoros or "Congo Brazzaville" or Zaire or "Congo Democratic Republic").af. OR ("Ivory Coast" or "Cote d "Ivoire" or Djibouti or "Equatorial Guinea" or Eritrea or Ethiopia or Gabon or "The Gambia" or Gambia or Ghana or Guinea or Guinea\$Bissau or Kenya or Lesotho or Liberia).af. OR (Madagascar or Malawi or Mali or Mauritania or Mauritius or Mozambique or Namibia or Niger or Nigeria or Reunion or Rwanda or "Sao Tome" or Principe or Senegal).af. OR (Seychelles or "Sierra Leone" or Somalia or "South Africa" or "South Sudan" or Sudan or Swaziland or Tanzania or Togo or Uganda or "Western Sahara" or Zambia or Zimbabwe).af.

AND

(Income or livelihood or gender or education or perception or attitude or deforestation or degradation or reforestation or regrowth or "biodiversity conservation" or "species loss" or "species gain" or "ecosystem services"). af.1156 results $\rightarrow 1152$ following duplicate removal

Received: 8 June 2016 Accepted: 5 January 2017

Published online: 13 February 2017

\section{References}

1. Kebede E, Kagochi J, Jolly CM. Energy consumption and economic development in Sub-Sahara Africa. Energy Econ. 2010;32:532-7.

2. Schure J, Levang P, Wiersum KF. Producing woodfuel for urban centres in the Democratic Republic of Congo: a path out of poverty for rural households? World Dev. 2014;64(Suppl 1):80-90.

3. liyama M, Neufeldt H, Dobie P, Jamnadass R, Njenga M, Ndegwa G. The potential of agroforestry in the provision of sustainable woodfuel in subSaharan Africa. Curr Opin Environ Sustain. 2014;6:138-47.

4. Karekezi S. Disseminating renewable energy technologies in sub-Saharan Africa. Annu Rev Energy Environ. 1994;19:387-421.

5. Bailis R, Ezzati M, Kammen DM. Mortality and greenhouse gas impacts of biomass and petroleum energy future in Africa. Science. 2005;308:98-103.

6. Girard P. Charcoal production and use in Africa: what future? Unasylva. 2002;211:30-3.

7. Brew-Hammond A, Kemausuor F. Energy for all in Africa - to be or not to be? Curr Opin Environ Sustain. 2009;1:83-8.

8. World Bank. Wood-based biomass energy development for Sub-Saharan Africa: issues and approaches. The International Bank for reconstruction and development. Washington, D.C. 20433; 2011.

9. Openshaw K. Biomass energy: employment generation and its contribution to poverty alleviation. Biomass Bioenergy. 2010;34:365-78.

10. Chidumayo EN, Gumbo DJ. The environmental impacts of charcoal production in tropical ecosystems of the world: a synthesis. Energy Sustain Dev. 2012;17:86-94

11. Kammen DM, Lew DJ. Review of technologies for the production and use of charcoal. Renewable and Appropriate Energy Laboratory report. Energy and Resources Group \& Goldman School of Public Policy. Berkeley: University of California; 2005. 
12. Smith KR. Health impacts of household fuelwood use in developing countries. In Forests and human health: Food and Agriculture Organization of the United Nations Rome. Unasylva. 2006;224:41-4.

13. WHO. Fuel for life-household energy and health. World Health Organization; 2006. http://www.who.int/indoorair/publications/fuelforlife/en/. Accessed 8 Apr 2015.

14. Cerutti PO, Sola P, Chenevoy A, liyama M, Yila J, Zhou W, Djoudi H, Atyi RE, Gautier D, Gumbo D, Kuehl Y, Levang P, Martius C, Matthews R, Nasi R, Neufeldt H, Njenga M, Petrokofsky G, Saunders M, Shepherd G, Sonwa D, Sundberg C, van Noordwijk M. The socioeconomic and environmental impacts of wood energy value chains in Sub-Saharan Africa. A systematic map protocol. Environ Evid. 2015;4:12. doi:10.1186/s13750-015-0038-3.

15. liyama M, Neufeldt $H$, Dobie P, Hagen R, Njenga M, Ndegwa G, Mowo J, Kisoyan P, Jamnadass R. Opportunities and challenges of landscape approaches for sustainable charcoal production and use. In: Minang PA, van Noordwijk M, Freeman OE, Mbow C, de Leeuw J, Catacutan D, editors. Climate-smart landscapes: multifunctionality in practice. Nairobi: World Agroforestry Centre (ICRAF); 2015. p. 195-209.

16. Randolph JJ. Online kappa calculator [Computer software]. 2008. http:// justus.randolph.name/kappa Accessed 13 Apr 2016.
17. Wallace BC, Small K, Brodley CE, Lau J, Trikalinos TA. Deploying an interactive machine learning system in an evidence-based practice center: abstrackr. Proc. of the ACM International Health Informatics Symposium (IHI). 2012. p. 819-824.

18. Arnold JEM, Köhlin G, Persson R. Woodfuels, livelihoods, and policy interventions: changing perspectives. World Dev. 2006;34:596-611.

19. IEA. Energy and poverty. In: International Energy Agency, editor. Paris: World Energy Outlook; 2002. http://www.worldenergyoutlook.org/ media/weowebsite/2008-1994/weo2002_part1.pdf Accessed 13 Apr 2016.

20. Gazull L, Gautier D. Woodfuel in a global changes context, in Wiley interdisciplinary reviews. Energy Environ. 2014. doi:10.1002/wene.115.

21. Ellis PD. The essential guide to effect sizes: statistical power, meta-analysis, and the interpretation of research results. Cambridge: Cambridge University Press; 2010

22. Grégoire G, Derderian F, Le Lorier J. Selecting the language of the publications included in a meta-analysis: is there a tower of babel bias? J Clin Epidemiol. 1995:8:159-63.

\section{Submit your next manuscript to BioMed Central and we will help you at every step:}

- We accept pre-submission inquiries

- Our selector tool helps you to find the most relevant journal

- We provide round the clock customer support

- Convenient online submission

- Thorough peer review

- Inclusion in PubMed and all major indexing services

- Maximum visibility for your research

Submit your manuscript at www.biomedcentral.com/submit 